\title{
ITERATION, FOUNDATION, AND REFLECTION
}

ZFC has become in its first order axiomatization the accepted set theoretic standard. We take, as usually done, as $\mathbf{Z}$ the system containing the Axioms of Extensionality, Pairing, Powerset, Sums, Separation and Infinity. ZF adds Foundation and Replacement, like Separation an axiom schema. ZFC adds the Axiom of Choice. ${ }^{6} \mathbf{Z F C}+\mathbf{G C H}$ adds the Generalized Continuum Hypothesis to $\mathbf{Z F C}$ - and so on for stronger axioms.

The antinomies (like Russell's Paradox ${ }^{7}$ ) are often taken as showing that Naïve Comprehension

$$
\begin{array}{ll}
\left(\mathrm{NC}_{1}\right) & (\exists \mathrm{y})(\forall \mathrm{x})(\mathrm{x} \in \mathrm{y} \equiv \varphi(\mathrm{x})) \\
\left(\mathrm{NC}_{2}\right) & (\forall \mathrm{F})(\exists \mathrm{y})(\forall \mathrm{x})(\mathrm{x} \in \mathrm{y} \equiv \mathrm{F}(\mathrm{x}))
\end{array}
$$

6 Historically this is misleading as Zermelo included the Axiom of Choice in his system, where he used it to prove well-ordering (in 1908). He also has an extra axiom for the empty set, $\varnothing$, but as in FOL the domain cannot be empty, one does not need this axiom, but gets $\varnothing$ by separation. In the 1920s Fraenkel and von Neumann and Skolem added Replacement. Zermelo's original system did not contain Foundation, but his system of 1930 does. His 1930 system ZF' leaves out the Axiom of Infinity as he then considered it to be an extra-logical existence assumption. Zermelo's formulation was not confined to FOL, but Skolem's clarification of 'definite' property as used in an instance of Separation led to first order ZFC. Cantor already stated and used both the Axiom of Choice and Replacement.

7 'Antinomy' will be used for a contradiction provable given some theory and its logic. A 'paradox' is just a theorem contrary to our expectations and prejudices. Already Zermelo stressed the importance of this distinction, as otherwise one sees the likes of too many antinomies where there are only paradoxes. Unfortunately usage is not so clear nowadays. By the way: The antinomy unfortunately called 'Russell's Paradox' was discovered some years earlier by Zermelo. It leads back - as many antinomies - to negative self-application of a property/predicate, the idea behind the canonical proof of Cantor's Theorem, which served as the context of discovering 'Russell's Paradox'. 
is wrong. The assumption that every concept $/$ property $^{8}$ has an extension, which is a set, is considered rejected. The first order axiom schema $\left(\mathrm{NC}_{1}\right)$ or the second order axiom $\left(\mathrm{NC}_{2}\right)$ are sometimes called 'naïve set theory'. They were by no means present in all approaches to set theory introduced in the $19^{\text {th }}$ century. Cantor's original set theory was concerned with combinatorial multiplicities. At times, though, he considered sets as 'united by a rule', which sounds like Comprehension. Comprehension was certainly present in the logicist approach to set theory of Frege and Russell.

Now, take a version of Comprehension: the Russell Set, defined as $R=\{x \mid$ $\mathrm{x} \notin \mathrm{X}\}$, and taking ' $\mathrm{x} \notin \mathrm{X}$ ' as the open formula $\varphi(\mathrm{x})$ or the property $\mathrm{F}$ yields the famous antinomy: $R \in R \wedge R \notin R$. The defining property of NOT BEING A SELF-MEMBER seems to violate the constitutive assumption behind Naïve Comprehension by not having an extension, on pains of inconsistency.

There is another reading of Russell's Paradox, however. Proceeding to Zermelo's Aussonderungsaxiom (Axiom of Separation) ${ }^{9}$ or not-naïve Comprehension scheme (of set theory $\mathbf{Z}$ )

(AS) $\quad(\forall \mathrm{x})(\exists \mathrm{y})(\forall \mathrm{w})(\mathrm{w} \in \mathrm{y} \equiv \mathrm{w} \in \mathrm{x} \wedge \varphi(\mathrm{x}))$

the property NOT BEING A SELF-MEMBER can be used to derive:

(NU) $\quad \neg(\exists \mathrm{x})(\forall \mathrm{y})(\mathrm{y} \in \mathrm{x})$

8 In the context of this essay I take "concept" and "property" to be synonymous within set theories, as is usually done. In (natural language) semantics concepts may be said to refer to properties, which are often not taken as sets. Set theoretic ontology is less fine grained. A distinction is made between formulas expressing a concept/property and the concept/property. CAPITALIZATION is used to signal a concept/property. Reflecting on set theory and its relation to our cognition concepts (like the concept SET) are taken in their usual sense as cognitive, and whether they are captured and explicated by a theory (say, of 'sets') is the matter of debate.

To be precise: It is a schema in the wff $\varphi$. Any set can be separated by this axiom schema which corresponds to a wff in the language of the theory. The constructible universe L (used in Gödel's relative consistency proof for the Axiom of Choice and the Continuum Hypothesis) consists only of such sets, which requires restricting the powerset operation to constructible subsets. 
the denial of a universal set. ${ }^{10}$ What Russell's Paradox shows on this reading is that the assumption of the existence of a universal set is illicit. Cantor's Theorem establishes that the powerset $\wp(\mathrm{x})$ of a set $x$ has a larger cardinality than $x$. Cantor's classical proof refutes the supposition of a bijection $\mathrm{f}$ between $x$ and $\wp(\mathrm{x})$ by considering the subset $\{\mathrm{x} \mid \mathrm{x} \notin \mathrm{f}(\mathrm{x})\}$. If $x$ is the universal set this naturally introduces the Russell Set (being an element and a subset of the universal set). The idea of a universal set thus stands in tension to a core ingredient of the concept SET: that every set has subsets, which should be collectible. " $\subseteq$ " is as central to set theory as " $\in$ ": one of them provides a sufficient foundation:

$$
\begin{array}{ll}
(\mathrm{D} \subseteq 1) & \mathrm{x} \subseteq \mathrm{y} \stackrel{\text { def }}{=}(\forall \mathrm{z})(\mathrm{z} \in \mathrm{x} \supset \mathrm{z} \in \mathrm{y}) \\
(\mathrm{D} \in 1) & \mathrm{x} \in \mathrm{y} \stackrel{\text { def }}{=}\{\mathrm{x}\} \subseteq \mathrm{y}
\end{array}
$$

The Powerset Axiom focussing on " $\subseteq$ ", therefore, deserves a special role in any set theory, as Comprehension and/or Separation focus on " $\in$ ". That $\mathrm{U} \in \mathrm{U}$ may seem less unnatural than $\wp(\mathrm{U}) \subseteq \mathrm{U}$ and $\wp(\mathrm{U}) \in \mathrm{U}^{1{ }^{11}}$

Comprehension is fine as long as we restrict the domain of objects to be comprehended. If we assume that there is no universal set or domain even Naiive Comprehension need not lead to the antinomies, as one cannot take for granted that $\mathrm{R}$ (or a similar cause of trouble) belongs to the objects (sets) to be comprehended. (AS) provides the safe formalization of this idea. The property NOT BEING A SELF-MEMBER can be taken as having an extension now that (AS) has been adopted. Any property has an extension relative to a base set. And if $a$ is the base set for an instance of (AS) with ' $\mathrm{x} \notin \mathrm{X}$ ', the extension of the subset corresponding to NOT BEING A SELF-

10 Proof (Outline). Assume $U$ exists. Take $U$ as the base set $x$ in (AS). The first conjunct on the right side of the biconditional can then be eliminated, being logically true. One arrives at the form of $\left(\mathrm{NC}_{1}\right)$ and the usual reasoning to the Russell Paradox goes through. Reject the existence assumption concerning $U$ by arriving at the contradiction. This proof can already be carried out in a weak subsystem of $\mathbf{Z}$, like Kripke-Platek set theory KP. Membership can hardly be indeterminate for a set theoretic realist. Even if this had some plausibility for some sets, with respect to $U$ something is in the universe or is not. Avoiding the Russell Paradox by banning $\mathrm{R}$ from $\mathrm{U}$ leaves us with the mystery where to put $\mathrm{R}$ then, or with the option that some collections cannot be sets, which leads to a set/non-sets distinction, we will look at in chapter II. Indeterminate membership plays no role here; theories without tertium non datur will be considered in chapter $\mathrm{V}$, but giving up tertium non datur may mean rejecting both $\mathrm{R} \in \mathrm{R}$ and $\mathrm{R} \notin \mathrm{R}$.

11 Even the problem with Frege's 'basic law' (V) goes back to this, since Frege at the same time defines extensions as objects (i.e. first order entities) and puts them in basic law $(\mathrm{V})$ in correspondence to courses of values (predication) of concepts (i.e. second order entities), by Cantor's Theorem there have to be more extensions of concepts (namely sets of objects) than objects (cf. Boolos 1998, pp.135-54). Because of the complete absence of a Powerset Axiom we do not consider set theories like KP (Kripke/Platek set theory) in detail. 
MEMBER relative to $a$ is $a$ itself (as by the Foundation Axiom no set is a member of itself, so that all members of $a$ satisfy the condition $\mathrm{x} \notin \mathrm{x}$ ).

The discovery behind the set theoretical antinomies then consists not in a claim about properties

(NNC) Not every property has an extension.

but in a claim about universality

(NU') There is no universal set.

Both claims are ontologically substantial and surprising. Hilbert, for instance, thought that conception formation was in trouble, as the idea that being able to determine whether something falls under a concept does not suffice for the concept's existence.

The argument against U works with Separation. Using (NC) leads to the antinomy. One reading of the antinomic argument can also be that it uses the assumption that the Russell Set $R$ is part of 'all' objects (i.e. within the range of " $\forall$ "). The range of " $\forall$ " on pains of contradiction thus cannot be universal, $R$ lying outside of it. Thus there is no unrestricted quantification over all collections. If " $\forall$ " ranges over all sets, $R$ cannot be a set after all. The collection of non self-membered sets turns out to be the range of " $\forall$ " in $\mathbf{Z}$ because of the Axiom of Foundation (i.e. turns out to be the iterative hierarchy $\mathrm{V}$ itself)! In this reading of the antinomic argument again $a$ set of all sets is excluded. The reasoning poses two problems we come back to again and again: (i) (NC) still allows building the forbidden collections $U$ and $R$, and (ii) the reasoning invites our naïve bewilderment where some collection is 'to be' when outside of the range of " $\forall$ ".

The naturalness of the idea of universality or a universal set may be related to the Calculus of Classes (cf. e.g. Hilbert/Ackermann 1928, Chap. 2). ${ }^{12}$ Textbooks unhesitatingly speak of a 'universal class' here. The Calculus of Classes systematizes our reasoning with respect to 'classes' of arbitrary objects by defining cuts, unions etc. The complement of such a 'class' $a$ is an absolute complement $\bar{a}$, such that $a \cup \bar{a}$ is the 'universal class'. The crucial point is that these 'classes' of the Calculus of Classes only contain individuals of the considered domain. There are no 'classes of classes'. The 'universal class' is just the domain considered. The 'classes' of the Calculus of Classes are neither sets nor classes. They obey some axioms (like Extensionality), but others (like Powerset) do not apply here. The concept SET exhibits much more complexity than the concept COLLECTION OF INDIVIDUALS!

12 In the following paragraph "class" is scare-quoted to make clear that these collections are not proper classes, but collections of individuals. 
Given the logical apparatus of $\mathbf{Z}$ we can even derive: $\mathrm{U}=\{\mathrm{x} \mid \mathrm{x}=\mathrm{x}\}=\varnothing$, even though we have: $(\forall \mathrm{x})(\mathrm{x}=\mathrm{x}) !^{13}$

There are several reasons why there is no universal set in $\mathbf{Z F}$ :

1. There is no U because this contradicts Cantor's Theorem (i.e. because of the Axiom of Powerset). For $U$ we should have $\wp(U) \subset U$, but this contradicts Cantor's Theorem (as, trivially, a subset has at most the cardinality of the superset). [By the way: Hilbert had a similar argument working with self-mappings of functions of numbers.]

2. There is no $U$ because this contradicts the Axiom of Foundation. For $U$ we should have $U \in U$ against Foundation.

3. There is no $U$ by the Axiom of Separation, as shown above.

4. As, because of further antinomies, there cannot be a set of all cardinal numbers or of all ordinal numbers - as was already clear to Cantor there can be no U, which had to contain these sets as separable subsets.

5. There is no $U$ by the Axiom of Pairing in combination with Foundation as $\{U\}$ could be built by Pairing (i.e. $U$ and $U$ again gives $\{U, U\}=\{U\}$ ), but $\{U\} \in U$ contradicts Foundation as $\{U\}$ does not have an element that does not share an element with it (as $U \in U)$.

The absence of a universal set yields more consequences in $\mathbf{Z , ~} \mathbf{Z F}$ and ZFC. In Z, ZF and ZFC absolute complements are missing: since subsets are separated relative to a base set the complement to a set $x$ is not the collection of all things not in $x$, but only the collection of those things in the base set which are not in $x$. This follows the spirit of Separation, but violates, supposedly, our intuition as to complements. Just as Comprehension is restricted in $\mathbf{Z}$ so is complement building. There cannot be absolute complements as the absolute complement to $\varnothing$ had to be $\mathrm{U}$.

As $\mathbf{Z F}$ and $\mathbf{Z F C}$ are naturally understood by the iterative hierarchy [cf. below] their definition of number cannot be Frege's. Frege used a flat universe and defined a cardinal number as the equivalence class of sets with the same equinumerosity - or a representative of that equivalence

13 Proof (Outline). If one allows for definition by abstraction in a pure set theory (i.e. without atoms, which are not sets) one has to use a scheme like the following:

$$
\{\mathrm{x} \mid \varphi(\mathrm{x})\}=\mathrm{y} \equiv((\forall \mathrm{x})(\mathrm{x} \in \mathrm{y} \equiv \varphi(\mathrm{x}) \wedge(\exists \mathrm{w})(\mathrm{w}=\mathrm{y})) \vee(\mathrm{y}=\varnothing \wedge \neg(\exists \mathrm{w})(\forall \mathrm{x})(\mathrm{x} \in \mathrm{w} \equiv \varphi(\mathrm{x})))
$$

Now, for an instance of this scheme with $U=\{x \mid x=x\}$, assume $U \neq \varnothing$, then the second disjunct on the right hand side is false. Therefore the first disjunct has to be true. This leads to contradiction again, by the proof for (NU). Thus the assumption has to be rejected. [In a set theory with atoms the second conjunct in the first disjunct has to be the meta-linguistic assumption that $y$ is a set, cf. Suppes 1960, p.34.] 
class. ${ }^{14}$ Frege defined equinumerosity by means of bijective functions. This cannot be done in $\mathbf{Z F}$ as, for instance, there are singletons of any rank in the hierarchy, so the supposed set representing 1 had to contain elements from any rank, but this is impossible for a set (contradicting the Reflection Principle): Sets have a minimal rank, the rank at which all their elements are present. A collection of sets of arbitrary high rank cannot be a set, and this cannot be or represent a number.

The idea that there is no universal set seems to go against our logical intuitions as we have developed them working with quantificational logics: There is always a domain of all objects to be quantified over.

What then can be the semantics of $\mathbf{Z}$ ? How are its quantifiers to be understood? Although there is no universal set, there is universal quantification in $\mathbf{Z}$. The axioms witness this. The Axiom of Separation, for instance, says of all sets that for any condition the corresponding subset exists. In terms of the iterative hierarchy [cf. below] the axiom talks about sets of any rank.

One issue should be made clear at the very beginning: The metaphors usually employed when setting out 'the construction' of some sets, say of the transfinite ordinals, should not be taken literally as involving some temporal procession of arriving at ever larger ordinals, ranks or cardinalities. As sets are abstract entities they do not depend in their existence on any one - not even God - counting up to them. Sets are simple there. All of them are there. The metaphors of construction merely serve to express the structures the sets employ, and may serve, sometimes, as didactic devices how we come to understand some set on the basis of another collection of sets. Thus, that there is no highest rank in ZFC should not be misunderstood as the set theoretic hierarchy $\mathrm{V}$ being under construction. All sets are there, thus $\mathrm{V}$ is there. For this ontological thesis and corresponding universal quantification it is irrelevant whether we have epistemic means to distinguish that totality from any incredibly large, but not total collection/set.

Like FOL, which does not count its domain to be one of the objects to be quantified over, $\mathbf{Z}$ itself need not talk about its domain. A stronger metalanguage may be used to model the semantics of $\mathbf{Z}$, typically a second order logic (SOL) talking about proper classes, one of which may be the domain of $\mathbf{Z}$. We come back to this later. ${ }^{15}$ But suppose there to be such a

14 In fact, in Frege's consistent system behind the Grundlagen der Arithmetik the concept BEING-IDENTICAL-TO-ONESELF should have an extension, and thus a number: the number of all things! The system can, however, not tell us what number this is (cf. Boolos 1987).

15 I use "set" to talk about sets and "class" to talk about proper classes (so called because these classes either are not sets or have no corresponding set, both usages are common, we come back to the idea of "correspondence' below). "Set" and "class" are 
model for $\mathbf{Z}$. What should the domain of it be called? It certainly looks like a universal set, as it comprises all sets. Then $\mathbf{Z}$ cannot be complete, since it does not deal with all collections of objects/sets. But wasn't it supposed to be complete in its application? V has to be a collection of sets, and can be no set itself in Z Z. Zermelo (cf. 1908) recognized this and concluded from the reasoning about the Russell Set that the domain of set theory 'is not itself a set'. There seem to be totalities beyond sets then.

$*$

The standard picture of the realm of sets accompanying $\mathbf{Z F}$ and $\mathbf{Z F C}$ is, at least nowadays, the iterative or cumulative hierarchy. It can be argued that Cantor had already a conception of sets congenial to this picture, because Cantor thought of sets as build by the iterative application of set building functions. Frege's set theoretic universe, in contrast, has to be conceived as flat (non hierarchic). The hierarchy was clearly developed by von Neumann (1929), wherefore it is sometimes called "von Neumann hierarchy". Zermelo developed a similar picture in the late 1920s. The Axiom of Foundation and the Axiom of Replacement determine this picture. Foundation expresses the idea that a set occurs at some earliest level in the hierarchy (as sets are build/defined by iteration of set building operations there is some - though possibly transfinite - number of preceding set building operations). As mentioned before, talk of 'building' sets should not be taken as a process of construction, but only as an easy way to express structural dependencies between sets all being already there. The Axiom of Replacement expresses the continuation of ever higher levels (e.g. by collection a transfinite sequence of iterations of applying the powerset operator into a single set).

In the pure version of the hierarchy the starting level (or 'rank') $V_{0}$ is $\varnothing$, then there are two ways of proceeding to higher ranks

$$
\begin{array}{ll}
\mathrm{V}_{\alpha+1}=\wp\left(\mathrm{V}_{\alpha}\right) & \text { for successor ordinals } \alpha \\
\mathrm{V}_{\delta}=\cup\left\{\mathrm{V}_{\alpha} \mid \alpha<\delta\right\} & \text { for limit ordinals } \delta
\end{array}
$$

the set theoretic universe $\mathrm{V}$ can then be seen as a hierarchy where later sets depend on preceding sets (although, of course, not in a temporal manner). The hierarchy is iterative as the two hierarchy building operations are applied over and over again. The hierarchy is cumulative as the sets present

thus not taken as synonymous here. All claims and theories referred to are adapted to this usage; formalisms/symbols are also rendered into the common format used here. Following ordinary usage equivalence sets are called "equivalence classes" although they are no classes. 
at $\mathrm{V}_{\alpha}$ are also present at all levels $\mathrm{V}_{\delta}$ with $\alpha<\delta .{ }^{16}$ Each set has some earliest rank of occurrence. All ranks are transitive sets (i.e. contain all members of members of members...). The strength of the operation of collecting the powerset provides the plenty of the next stage. Reflections about how strong the idea of a powerset is concern directly the issue of the Generalized Continuum Hypothesis (GCH).

The picture is slightly different in a set theory with urelements. The set of urelements $\mathrm{M}$ lays at the foundation of the hierarchy $\mathrm{V}_{0}=\mathrm{M}$. The two ways of proceeding are accompanied by the requirement that for each $\mathrm{V}_{\alpha}, \mathrm{M} \subseteq$ $\mathrm{V}_{\alpha}$. A corresponding set theory needs to distinguish sets from non-sets and is called $\mathbf{Z F U}$ or $\mathbf{Z F C U}$. $^{17}$

16 Remember that $\varnothing \subseteq \mathrm{V}_{\alpha}$ for any $\mathrm{V}_{\alpha}$ as $\mathrm{V}_{\alpha}$ is a set. Thus at $\mathrm{V}_{\alpha+1} \varnothing$ and $\{\varnothing\}$ are present and thus each stage contains all preceding stages.

17 Usually the system is called $\mathbf{Z F U}$, with $U$ being the set of urelements. The name "ZFU" may thus confuse in the context of our investigation into the existence of the universal set U. Nonetheless we stick with the usual name "ZFU" as urelements and thus ZFU and ZFCU play no vital role in this book. For us it is important to distinguish the set of all sets U from the class of all sets V, so we need the name "U" in addition to "V". 
Thus one can picture $\mathrm{V}$ as either a pure hierarchy of $\mathbf{Z F}, \mathbf{Z F C}$ (upper part in the picture) or a hierarchy based in domain of non-sets (lower part).
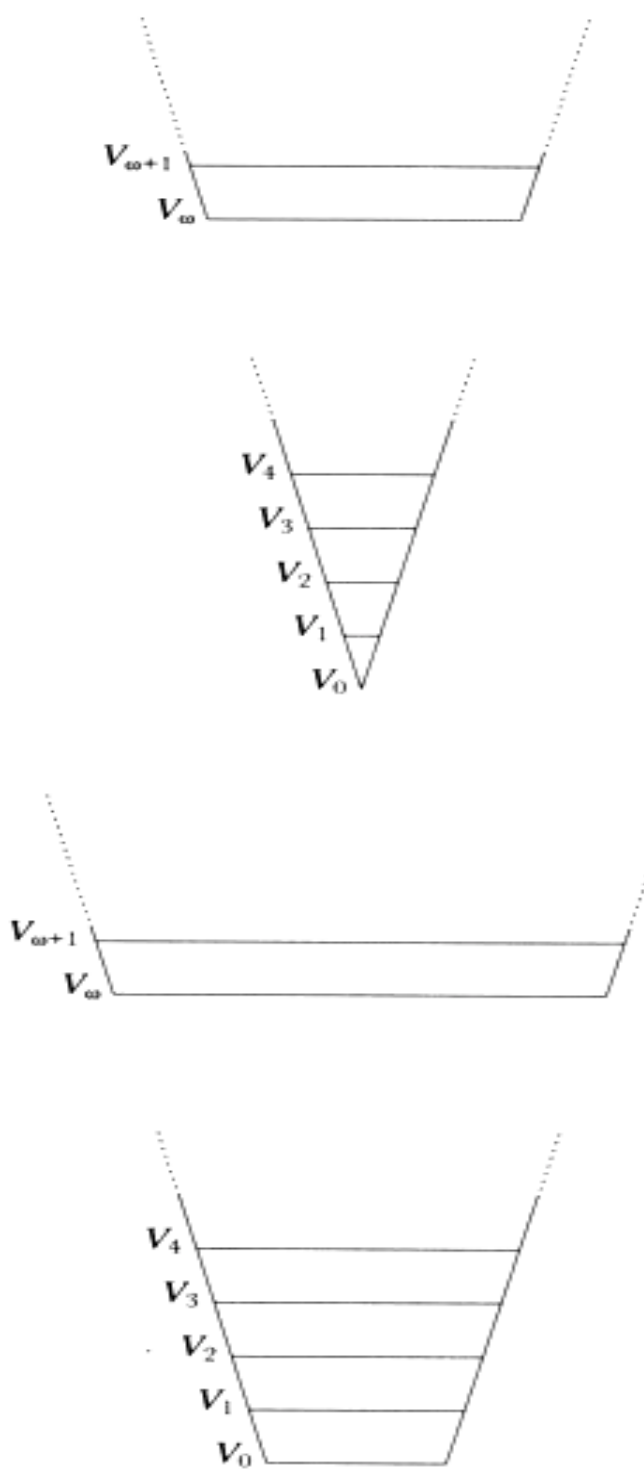

ZFU has a broader base than $\mathbf{Z F}$. The dots before $\mathrm{V}_{\omega}$ indicate that $\mathrm{V}_{\omega}$ is the first limit level (of transfinitely many).

$\mathbf{Z}$ takes us with the Axiom of Inifinity to $\mathrm{V}_{\omega}$, but not to arbitrary high ranks in V. We need $\mathbf{Z F}$ (i.e. Replacement) to go further. By Replacement we know that the function in $n$ for $n \in \omega$ which takes as value the $n$-time powerset of $\omega$ has as range $a$ set, since $\omega$ is a set (by the Axiom of Infinity). Therefore (by the Axiom of Union) the union of all these powersets exists as $a$ set, and thus as a next rank in V. Now we can move in ZF beyond $\mathrm{V}_{\omega+\omega}$. Note also that in this rank all other $\mathbf{Z F}$-axioms are satisfied, while - 
by Foundation - the rank is not a member of itself, which establishes the independence of Replacement from the other $\mathbf{Z F}$-axioms. ${ }^{18}$

$\mathrm{Up}$ to $\mathrm{V}_{\omega}$ we find in pure set theory the hereditarily finite sets. They fit naturally to defining the ordinals in von Neumann's way: $\mathrm{n}+1 \stackrel{\text { asf }}{=} \mathrm{n} \cup\{\mathrm{n}\}$ and take $\varnothing$ as 0 . Then in $V_{\omega}$ a transitive set of transitive sets is a number. We get: $\mathrm{n} \in \mathrm{V}_{\mathrm{n}+1}, \mathrm{n} \notin \mathrm{V}_{\mathrm{n}}, \mathrm{V}_{\mathrm{n}} \in \mathrm{V}_{\mathrm{n}+1}, \mathrm{~V}_{\mathrm{n}} \subseteq \mathrm{V}_{\mathrm{n}+1}$. Ranks and numbers thus are $\in$ ordered. The hereditarily finite sets fulfil the axioms of ZFC save the Axiom of Infinity, although the Axiom of Choice and the Axiom of Replacement become unimportant here: The Axiom of Infinity is thus independent from the other ZFC-axioms. The finite system is sometimes called: $\mathbf{Z F C}^{-\infty}$. In fact one could add an Axiom of Finiteness here:

$$
\neg(\exists \mathrm{x})(\varnothing \neq \mathrm{x} \wedge(\forall \mathrm{y})(\mathrm{y} \in \mathrm{x} \supset \mathrm{y} \cup\{\mathrm{y}\} \in \mathrm{x}))
$$

Obviously the Axiom of Finiteness is true up to $V_{\omega}$, i.e. for all hereditarily finite sets. And equally obviously $\mathrm{V}_{\omega}$ (i.e. the domain of that theory) is not finite. We meet the same situation as with Quine's basic finite arithmetic [in chapter III]. Even $\mathbf{Z F C}^{-\infty}$ can do what Peano Arithmetic, PA, does: prove theorems concerning representability and provability (e.g. Tarski's and Gödel's theorems. ${ }^{19}$ Note that the hereditarily finite sets provide an intended model for $\mathbf{Z F C}{ }^{-\infty}$ (i.e. in contrast to other unintended countable models for ZFC). Note also - and this may be thought to be important that Naïve Comprehension causes no trouble within the hereditarily finite sets. The Russell Set, for instance, does not exist up to $\mathrm{V}_{\omega}$ as it contains all hereditarily finite sets, since they satisfy Foundation, and thus is infinite. If the set of urelements is finite as well - as one may expect in a finite physical universe - this finite consistency of Naïve Comprehension may be the background of our intuitive support of Naïve Comprehension. Let us note this as a theorem (" $y$ " not occurring in $\varphi$ as always):

(FNC)

$$
|\{\mathrm{x} \mid \varphi(\mathrm{x})\}|<\aleph_{0} \supset(\exists \mathrm{y})(\forall \mathrm{x})(\mathrm{x} \in \mathrm{y} \equiv \varphi(\mathrm{x}))
$$

18 Remember not to confuse the indices of ranks above $V_{\omega}$ with theses about the cardinality of the rank itself, the order type of its largest member or the index number occurring for the first time at that rank. $\omega+1$ (i.e. $\{2,3,4, \ldots 1\}), \omega+2, \omega+3$ etc. are, because they are order types (i.e. relational) subsets of $\omega \times \omega$, thus countable, thus sets of ordered pairs (i.e. given the usual definition of ordered pairs, sets of sets of sets of natural numbers) being subsets of $\mathrm{V}_{\omega+2}$, members of $\mathrm{V}_{\omega+3}$. These ranks have cardinality $\aleph_{2}, \aleph_{3}$ respectively and contain many, many ordinals. $\mathbb{R}$ under the usual construction (as a set of sets of sets of natural numbers) is a subset of $V_{\omega+2}$, member of $V_{\omega+3}$. $\mathbb{R}$ is uncountable, whether it has a order-type (not just a simple ordering, but a well-ordering) is not obvious and is ensured only by $\mathbf{Z F C}$, not $\mathbf{Z F}$.

19 Cf. Fitting 2007. The Peano/Dedekind-Axioms for the successor function and induction follow easily in $\mathbf{Z}$ from the Axiom of Infinity. Taking natural numbers as von Neumann ordinals makes obvious that 0 is no successor and that the successor relation is functional. Induction follows since an inductive property is inherited by the successor relation, thus contains $\omega$. 
Let us leave $\mathbf{Z F C}{ }^{-\infty}$ behind and look at all ranks in $\mathrm{V}$. With a little pretense we can say: In the iterative hierarchy exists at some rank any proper subset of $\mathrm{V}$, i.e. (a) pretending for the moment that the non-set $\mathrm{V}$ has subsets and (b) speaking only about collections that can be sets (excluding a set of ordinals etc.). We can approximate Naïve Comprehension up to an arbitrary rank: $\mathrm{y}=\{\mathrm{x} \mid \varphi(\mathrm{x})\}$ exists for any $\varphi$ as long as the rank of $y<\alpha$ for some ordinal $\alpha$. The set $y$ exists then somewhere below $\alpha$. We can say in general: If a set $x$ exists $x$ has some rank. ${ }^{20}$ Existential statements are, if true, true in parts of V. The Principle of Reflection correspondingly claims that if a general sentence or a finite collection of sentences in the language of $\mathbf{Z F C}$ is set theoretically true, there is a least rank $\mathrm{V}_{\alpha}$ which can serve as its model (with variables in the sentences bounded to rank $V_{\alpha}$ ). ${ }^{21}$ One might expect that as all specific sets mentioned in a sentence have a rank. Limit ranks ensure this structure. Once again - as with Naïve Comprehension - we seem to approximate talk of all sets! The Reflection Principle is equivalent to the Axiom of Replacement. ${ }^{22}$ So the fully developed picture of the iterative hierarchy established by Replacement approximates universal set theoretic talk. Unfortunately this would be too good to be true.

On the one hand we approximate universal set theoretic talk. And not just one may claim (as Kreisel 1967 did) - set theoretic talk: Set theory can be considered to be our strongest formal system, the system to be used in the meta-theory of all other systems. Then: If some claim in some informal system is intuitively valid and can be captured in some formal system it has a set theoretical model. Kreisel's Thesis so states: Whatever is valid is valid

20 Proof (Outline). If $x$ existed without a minimal rank at which it exists, $x$ would contain all ordinals as a subset, which is impossible.

21 This does, of course, not hold for an infinite collection of sentences as all infinitely many instances of the schema of Replacement enforce V. The Principle of Reflection is another reason why ZFC cannot be finitely axiomatized: If ZFC could be finitely axiomatized, then it would establish - by the Principle of Reflection - a model of itself, thereby establishing its own consistency, contradicting Gödel's Second Incompleteness Theorem.

22 Proof (Outline). The Reflection Principle entails Replacement, since if the antecedent of Replacement is true, there has to be a rank $V_{\alpha}$ modelling it; the set postulated as existing in the consequent of Replacement will be a subset of that modelling rank $\mathrm{V}_{\alpha}$. Replacement entails each instance of the Reflection Principle in going through the quantifiers of the finitely long compound $(\forall x) \varphi(x)$ taking the lowest possible rank of satisfying instances (which have to be there to make $\varphi(x)$ true) and uniting them and their dependencies (by a Replacement function) into a highest most comprehensive rank, which thus models $(\forall x) \varphi(x)$. Omitting the Replacement schema and restricting separation to formula $\varphi$ with quantifiers bounded to some set provides a

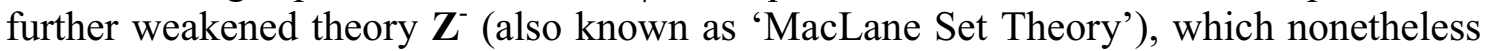
proves sufficient for most of mathematics. 
in a set theoretical model, and if - as we may suppose - finitely many sentences were used in that piece of reasoning, it is valid at some rank $\mathrm{V}_{\alpha}$.

On the other hand, however, we, obviously, shift the domain of reference from $\mathrm{V}$ to some rank $\mathrm{V}_{\alpha}$. So a universal statement (say, the Axiom of Pairing) does no longer talk of all sets, but only of those up to $\mathrm{V}_{\alpha}$. Seen in this light the Principle of Reflection resembles the Löwenheim/SkolemTheorem in allowing for non-standard or unintended models of universally quantified set theoretic sentences. As $\mathrm{V}_{\alpha}$ can be arbitrary high one may see this as less concerning than the countable models ensured by the Löwenheim/Skolem-Theorem. If $\mathrm{V}_{\alpha}$ is a sufficiently high transfinite rank we approximate universal talk. We can also understand the possible shift of domain of reference as underlining the insight that universal set theoretic talk is bound to strong axioms like Replacement.

The universe $\mathrm{V}$ is not reached by any ladder ('construction principle') used within it. It is as strongly inaccessible by such steps as it can be. Otherwise we only have a temporary halting point $\mathrm{V}_{\alpha} . \mathrm{V}$ is no number, is no set, no union or power of sets. V can only be thought as sui generis. How do we know this? Because otherwise it could be superseded in one of the usual ways. We thus have a transcendental argument concerning V's nature: it cannot be otherwise, since otherwise it wouldn't be.

Without the Axiom of Foundation or endorsing an Anti-Foundation Axiom the realm of sets is larger containing with the unfounded sets more collection like entities. Where are these collections collected in? U seems a good candidate for an collection of unfounded collections as $U \in U$ itself. But unfortunately $\mathbf{Z}$ forbids U. Are unfounded collections sets? Or does our concept SET entail that sets are grounded collections? In this case we had the problem that on the one hand we had to endorse the Axiom of Foundation, but this excludes $U$ from our set theory. If sets are abstract entities nothing seems to exclude that they contain themselves as all spatial images are inappropriate. Picturing non-wellfounded sets by graphs (cf. Aczel 1988) shows easily membership bending back to its origin. Antifoundationalist set theories contradict our concept of set, however, if set identity becomes more than identity of membership (cf. Aczel 1988, chap.4). The iterative hierarchy motivates our picture of sets as wellfounded by stressing the idea of ontological structural dependence between a set and its members. In this light a set containing only itself, $x=\{x\}$, seems unnatural. $U$, in contrast, contains besides all other things itself. We might recognize $U$ as a set sui generis and allow for $U$ what we do not allow for other sets. Foundation would make an exception for U. But the exceptions would not end here as $U$, being subject to the others axioms if still a set, is exceptional - even inconsistent - with respect to Cantor's Theorem, for instance. Foundation certainly is built in the iterative hierarchy and $\mathrm{V}$ does not pose the problems with respect to Foundation that 
U does. According to the story of the iterative hierarchy, unfounded sets do not exist. The Axiom of Foundation follows from the set up of the cumulative hierarchy. The two conditions to proceed to higher ranks ensure the axioms of Pairing, Sums, Powerset and Infinity. Coupled with the idea of sets being extensional the structural properties of the iterative hierarchy thus entail the ZF axioms (cf. also Boolos 1989).

There are - besides the question of an Anti-Foundation Axiom incompatible set theoretic axioms (like the Axiom of Choice $v s$. the Axiom of Determinacy ${ }^{23}$ ), which shows that there are related realms of set-like entities (sharing the basic axioms), but which cannot be consistently united. There might be a unified inconsistent realm of all these sets [cf. Chap. V]. Even the incompatibility need not show that our concept of set is not settled. One of the set theories may be thought to be more natural. Even a concept SET settled in its basic aspects (like set separation and powerset existence) may leave some questions unsettled. The (Generalized) Continuum Hypothesis is the best known example. The simple Continuum Hypothesis $\left[\neg(\exists \mathrm{x})\left(\aleph_{0}<|\mathrm{x}|<2^{\aleph o}\right)\right]$ is even independent of the Axiom of Choice. $^{24}$

$\mathrm{V}$ has sets of arbitrary high rank. V itself does not occur in the hierarchy itself. $\mathrm{V}$ taken as the proper class of all ranks in $\mathrm{V}$ is a model of $\mathbf{Z F}$. If $\mathrm{V}$ exists $\mathbf{Z F}$ is consistent, as $\mathrm{V}$ satisfies all its axioms. Large cardinals (strongly inaccessible cardinals beyond the reach of any set building

23 Cf. Jech 2003, pp.627-43. The Axiom of Determinacy in so-called 'Descriptive Set Theory' contradicts the Axiom of Choice, what one may take to be bad enough. It also entails some strange results for large cardinalities (like $\aleph_{1}, \aleph_{2}$ being measurable cardinals, but $\aleph_{3} \ldots$ not being measurable). ZFC seems closer to our conception of sets in this regard.

24 Proof (Outline). Alephs are defined as infinite well-orderable cardinals. The Axiom of Choice is equivalent to the statement that any infinite cardinal is an aleph (as it implies the Well-Order Principle). Negating the Axiom of Choice (and thus the WellOrder Principle) one may endorse the simple Continuum Hypothesis but maintain $2^{\text {No } \neq}$ $\aleph_{1}$, since one may now deny that the Continuum can be well-ordered, whereas the combination with the Axiom of Choice entails $2^{\aleph_{0}}=\aleph_{1}$, since the Axiom of Choice entails that any infinite initial ordinal is an aleph.

Cantor proved in 1883 that there is no cardinality between the cardinality of the collection of finite ordinals $\left(\aleph_{0}\right)$ and the cardinality of the collection of all countable well-orderings of $\omega$, that cardinality thus being the next well-orderable cardinality: $\aleph_{1}$. Given the Continuum Hypothesis $2^{\mathrm{No}}$ is the cardinality of all countable linear orderings of $\omega$. Given the Well-Order Principle thus $\aleph_{1}=2^{\text {ऽo }}$.

The Generalized Continuum Hypothesis $(\mathrm{GCH})$ entails the Axiom of Choice: Using the first aleph GCH claims for all infinite cardinals $\mathrm{x}=2^{\mathrm{y}+\aleph_{\mathrm{o}}} \cdot \mathrm{x}=2^{\mathrm{y}+\aleph_{\mathrm{o}}}$ entails $x$ being an aleph, which makes $y$ an aleph. The GCH thus excludes that there are cardinals in between well-orderable cardinals (i.e. in between well-orderable sets), so that via its respective cardinal number any set can be well-ordered. (On arguments and intuitions around CH and GCH cf. Potter 2004, pp.266-82; Maddy 1988, §2.) 
operation by being uncountable, regular and greater than $2^{\delta}$ for any preceding cardinal $\delta$ ), if existing, are such models as well. For $\mathrm{V}$ the axioms of $\mathbf{Z F}$ are construction principles and thus trivially satisfied. For (strongly) inaccessible cardinals the important observation is that they are assumed to be just larger transitive sets. Take the least such cardinal; any function within it is of lower rank as the cardinal itself; thus the range of the function is a set, which has this least inaccessible cardinal satisfy the Axiom of Replacement - the other axioms are obviously satisfied again (cf. Jech 2003, pp.165-67).

Having all subsets of a rank present at the next rank suits the Axiom of Choice: If a family of non empty sets $x$ exists at some rank $\mathrm{V}_{\alpha}$, the members $y$ of that family exist already at lower ranks $\mathrm{V}_{\delta}$ with $\delta<\alpha$, and their members $z$ exist already at lower ranks $\mathrm{V}_{\gamma}$ with $\gamma<\delta$ (relative to a $\delta$ for some $y$ ); thus as these $z$ are elements of some $\mathrm{V}_{\gamma}$ a set $w$ containing one of them for each $\mathrm{y} \in \mathrm{x}$ exists (at the latest) at the rank $\mathrm{V}_{\alpha}$ of $x$. Choice is natural in the iterative hierarchy. $\mathrm{V}$ rather corresponds to $\mathbf{Z F C}$.

Once we have one of the inaccessible cardinals or the class $\mathrm{V}$ of all sets we have a model of $\mathrm{ZF}$ and could be content with respect to our theory of sets. So should we care about their nature?

Leaving $\mathrm{V}$ to the side for a moment let us consider large cardinals. We have just talked about them, so we know something about their nature and we can ascribe properties to them. So they should be the objects of some theory.

Zermelo thought of strongly inaccessible cardinals (his 'Grenzzahlen') forming themselves an unbounded sequence. This, however, implies that we quantify over them, and are again in the situation of asking over what domain now our quantifiers run. Is this collection of Grenzzahlen itself some Grenzzahl? Supposedly not to avoid antinomies of the Burali-Fortitype. Then again if we now introduce Super-Grenzzahlen we can start all over again with them - and once more the whole process iterates. Zermelo thought: 'This series reaches no true completion in its unrestricted advance, but possesses only relative stopping-points, ...' (1930, p. 47).

Now, this way of thinking may be innocent for a constructivist, but for a set theoretic realist the idea that sets have to come into existence is simply wrong. Placing them at some rank in the hierarchy does not mean that they come later (in time?) than the other sets. Frege's universe is antifoundational. And for a Platonist an anti-foundationalist universe has the advantage of keeping all ideas of stepwise construction at bay. As all abstract objects are there they exhibit some ontological dependencies, but this does not require that some are before or beneath others. Impredicativity is no problem in such an anti-foundational universe. Zermelo himself 
rejects any spatiotemporal associations. A well-ordering 'has nothing at all to do with spatiotemporal arrangement'. He also thought the term "choice" to be problematic as one may associate (temporally) successive choices being performed, where we have only a representational/selectional correlation (cf. Ebbinghaus 2007, p.69, 135). The ranks express a structural dependency only. All ranks are there. In the same way all of that coming beyond the set theoretic ranks (i.e. any large cardinal) is there. Thus there should be a collection of it all. Assuming a sequence of large cardinals thus does not seem to solve the problem of collecting sets, but either adds the issue of an incomplete universe [cf. Chap. VI] or means that V contains them all and only our set theory, say $\mathbf{Z F C}$, is not complete yet and has to be strengthened by further axioms.

The issue of large cardinals is independent from that of the universe of sets. If one can argue that some idea of some type or large cardinals comes from our concept of sets - say, why should $\aleph_{0}$ be the last inaccessible? - then these large cardinals may be thought of as stages in $\mathrm{V}$ above those which ZFC (so far) treats of. Any type of closure operation on preceding collections should correspond to a set within V. This idea resembles the content of the Reflection Principle: Any finitely specified closure condition can be modelled by some rank. Large cardinals may provide a universe and a model for ZFC, but they differ from classes in being collectable themselves and thus being members of the overall universe of (extended) set theory. Another argument for such additional sets stems from Scott's proof that $\mathrm{V} \neq \mathrm{L}$ given large cardinals, as the notions of (unrestricted) powerset and uncountability stand in conflict to $\mathrm{V}=\mathrm{L}$. The constructible universe seems unnatural, even though $\mathrm{V}=\mathrm{L}$ entails the Axiom of Choice and the Generalized Continuum Hypothesis, excluding it speaks in favour of large cardinals. The constructible universe violates the idea of purely extensional sets inasmuch as pure extensionality should allow for sets beyond any descriptive powers. One might think that it follows the idea of Naïve Comprehension, that sets correspond to properties, but why should all objective properties correspond to formulas in the first place? Proceeding to the next rank by the full powerset operation suits the simple idea of the powerset. Curtailing the powerset to subsets which are definable leaves out sets that should be there.

$\mathrm{V}$ is the ultimate model of the universe also in the sense that constructions like 'forcing' or means of building 'inner models' start from V (cf. Arrigoni 2007; on the formal details cf. Jech 2003, pp.175-223). ${ }^{25}$

25 Leaving here to the side the problem that such models are non-standard or unintended, e.g. in being countable; cf. the remarks in the next chapter on limits of expressivity. One may add that inner models like L, which restrict the powerset operation, but satisfy the others axioms in their standard reading (relative to the shrunken universe), are less non-standard than models generated by forcing. 
The iterative hierarchy does not know several classes. It might be preferable not to call $\mathrm{V}$ a class, but to treat of $\mathrm{V}$ as a very special object in its own right - an issue of axiomatic ontology. If we call $\mathrm{V}$ a class it is not to be thought of in the manner of NBG or MK, since there is no part of set theory which addresses it, like Comprehension and Limitation of Size address classes in NBG or MK [cf. chap. II below]. V is not in the range of set theoretical quantifiers. It is not in the domain. Calling V 'a class' in the context of the iterative hierarchy and ZFC means there exists only one class (outside of our theory of sets). ${ }^{26}$

$\mathrm{V}$ is the range of the quantifiers in ZFC. Cantor claimed that every potential infinite presupposes an actual infinite 'and cannot be thought without it' (cf. Cantor 1887). This is the Domain Principle: Speaking of and quantifying the $x$ presupposes the domain of the $x .^{27}$

$\mathrm{V}$ is a very special entity, both within the picture of the iterative hierarchy as in our meta-theory modelling our theory of sets. $\mathrm{V}$ has no subsets as $\mathrm{V}$ is no set. V is not well-ordered - even in the presence of the Well-Order Principle only sets are well-ordered. V is not the domain of a (replacement) function, sets are - and so on. V contains all ordinals and all cardinals, but there is neither a set of all ordinals nor a set of all cardinals. They cannot be established as subsets of V, since V is no set (and thus Separation does not apply to it).

For $\mathrm{V}$ to be more than a stopping point to be superseded $\mathrm{V}$ has to be an entity sui generis. This means informally that $\mathrm{V}$ is exactly what the picture of the iterative hierarchy shows it to be. $\mathrm{V}$ is determined, not indefinite, and unique. Formally this means

- that V cannot be an element of whatsoever other collection - on pains of re-introducing distinctions of the set/class-type

- that there are no other entities of V's type (not a collection of proper classes)

- that $\mathrm{V}$ is an entity which can be talked about by its name, without including it into a domain of reference.

26 At some time Cantor considered distinguishing several 'absolutely' large, 'inconsistent' collections (like those of all ordinals or all cardinals). But they play no role in a transfinite set theory based on standard logic. Even apart from producing antinomies, these collections play no indispensable role in proofs about sets. So Cantor came to consider the single absolute, inconsistent totality beyond any further increase.

27 Cf. Moore 1990, pp. 114-22; Tiles 1989, pp. 95-107. The principle sometimes ignoring Cantor? - is discussed as 'All-in-one Principle', going back to (Cartwright 1994). 
$\mathrm{V}$ is not a standard object of (set theoretic) model theory. The only thing $\mathrm{V}$ 'does' is containing all the sets. A universally quantified sentence of pure set theory is meaningful as there is an entity which provides all the variable values: $\mathrm{V}$.

A unified language has to distinguish urelements, sets and V. Again: V cannot be unified with them in a domain. The name "V" refers to V rigidly. End of story. 
